\title{
Coupling of wave and circulation models in coastal-ocean predicting systems: a case study for the German Bight
}

\author{
Joanna Staneva, Kathrin Wahle, Heinz Günther, and Emil Stanev \\ Institute for Coastal Research, HZG, Max-Planck-Strasse 1, 21502 Geesthacht, Germany \\ Correspondence to: J. Staneva (joanna.staneva@hzg.de)
}

Received: 19 September 2015 - Published in Ocean Sci. Discuss.: 21 December 2015

Revised: 6 April 2016 - Accepted: 11 May 2016 - Published: 15 June 2016

\begin{abstract}
This study addresses the impact of coupling between wave and circulation models on the quality of coastal ocean predicting systems. This is exemplified for the German Bight and its coastal area known as the Wadden Sea. The latter is the area between the barrier islands and the coast. This topic reflects the increased interest in operational oceanography to reduce prediction errors of state estimates at coastal scales, which in many cases are due to unresolved non-linear feedback between strong currents and wind waves. In this study we present analysis of wave and hydrographic observations, as well as results of numerical simulations. A nestedgrid modelling system is used to produce reliable nowcasts and short-term forecasts of ocean state variables, including waves and hydrodynamics. The database includes ADCP observations and continuous measurements from data stations. The individual and combined effects of wind, waves and tidal forcing are quantified. The performance of the forecast system is illustrated for the cases of several extreme events. The combined role of wave effects on coastal circulation and sea level are investigated by considering the wavedependent stress and wave breaking parameterization. Also the response, which the circulation exerts on the waves, is tested for the coastal areas. The improved skill of the coupled forecasts compared to the non-coupled ones, in particular during extreme events, justifies the further enhancements of coastal operational systems by including wave effects in circulation models.
\end{abstract}

\section{Introduction}

In the last decade, the north European coasts were affected by severe storms which caused serious damages in the North Sea coastal zones. Additionally, different human activities, e.g. offshore wind power industry, oil industry and coastal recreation necessitate information about the sea state in the coastal ocean with high resolution in space and time. There seems to be a consensus that high-quality predictions of extreme events, like storm surges and flooding caused by storms, could substantially contribute to avoid or minimise human and material damages and losses. Therefore, reliable wave forecasts and long-term statistics of extreme wave conditions are of utmost importance for the coastal areas. In many coastal areas the need for reliable risk assessments increases the demand of precise coastal predictions. This cannot be achieved by further neglecting the wave-current interaction in coastal ocean operational forecasting.

Wave-current interaction is recently an important issue in the field of coastal ocean forecasting (Roland and Ardhuin, 2014; Bolaños et al., 2014). Understanding this process is of utmost importance on the road of fully integrating the atmospheric, wave and ocean models and their further coupling with biological, morphological and hydrographical forecasting systems. The uncertainties in most of the presently used models results from the non-linear feedback between the currents, water level variations and wind waves, which can no longer be ignored, in particular in the coastal zone. The joint impact of surges, currents and waves is strongly inter-related (Wolf et al., 2011; Brown et al., 2011) and those cannot be considered separately for coastal ocean predictions.

The ocean waves affect not only the sea level but also the currents and mixing, the latter being of utmost importance for the sediment dynamics (Lettmann et al., 2009). Prandle 


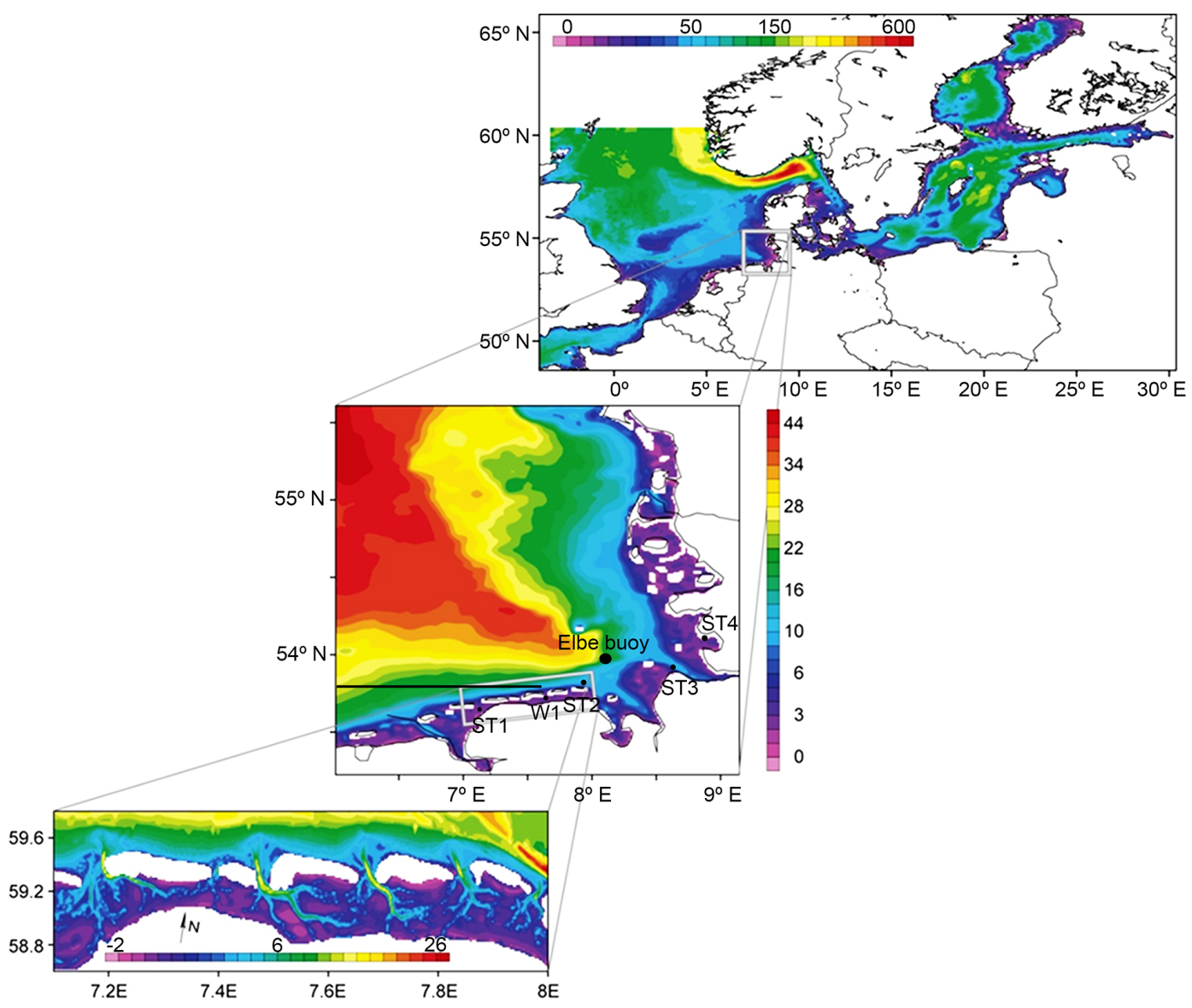

Figure 1. Nested grid model domains for the North Sea (top pattern), German Bight (middle pattern) and the east Wadden Sea (bottom pattern). The spatial resolution is $3 \mathrm{~nm}, 1 \mathrm{~km}$ and $200 \mathrm{~m}$, respectively. The geographical location of stations and sections analysed later are shown as well.

et al. (2000) demonstrated the need of accounting for surface waves with a significant wave height larger than one metre in the sediment modelling. This is of big importance for sediment dynamics and other ecosystem processes (Wolf and Prandle, 1999). These authors also showed the effects of waves due to surges and tides; on the other side the waves' characteristics are affected by the changes of sea level height due to tides and wind.

The main effects of waves that are commonly considered in the coupled modelling are due to radiation stress and Stokes drift. Babanin et al. (2010) showed that interaction of turbulence and bottom stress is also very important.

Wave-current interaction has been a topic of many studies recently (Ardhuin et al., 2008; Mellor, 2003, 2008, 2011; Kumar et al., 2012; Michaud et al., 2012; Zodiatis et al., 2015). Mellor $(2003,2005,2008)$ extended the radiation stress formulation based on the linear wave theory of Longuet-Higgins and Stewart (1964). Bennis and Ardhuin (2011) questioned the method of Mellor and suggested the use of Lagrangian mean framework leading to the so-called vortex force. Vortex force method has been implemented in ROMS-SWAN (Ku- mar et al., 2012; Lane et al., 2007; McWilliams et al., 2004; Uchiyama et al., 2010). Moghimi et al. (2013) critically compared the two approaches claiming that the radiation stress formulation showed unrealistic offshore directed transport in the wave shoaling regions; on the other hand the results of longshore circulations performed similarly for both methods. Aiki and Greatbatch $(2013,2014)$ proved that the radiation stress formulation of Mellor is applicable for small bottom slopes. Bolaños et al. $(2011,2014)$ demonstrated the importance of wave-current interactions in a tidally dominated estuary and showed that the inclusion of wave effects through 3 -D radiation stress improves the velocity in the study area. They also compared the different radiation stress methods and concluded that for the tidally dominated area the 3-D version of radiation stress produces better results than the 2-D version. Polton et al. (2005) found that accounting for the Coriolis-Stokes forcing results in encouraging agreement between model and measurements of the mixed layer. Janssen (2012) showed positive impact of wave breaking to the daily cycle of sea surface temperature. Later Breivik et al. (2015) demonstrated reduced bias between modelled and 
measured water temperature by incorporating the CoriolisStokes forcing, turbulence induced by breaking waves and ocean side stress in the NEMO model at global ocean scale. Weber et al. (2006) estimated that the wave induced stress is about $50 \%$ of the total atmospheric stress for moderate to strong wind. Wolff et al. (2011) studied the effects of waves on hydrodynamics; Brown et al. (2013) considered the wave effects on the storm surges; and Roland et al. (2009) studied wave effects on water level for the Adriatic Sea. The importance of ocean depth and velocity variations for the simulated waves in the estuaries is analysed by Pleskachevsky et al. (2011) and Lin and Pierre (2003). However, within the framework of practical coastal ocean forecasting, the interactions between waves and currents are still not yet enough considered.

In this study we will address the coupling between wave and circulation models for coastal ocean prediction systems with the example of the German Bight. We do not plan to analyse the role of different parameterisations used. Rather we will demonstrate the areas of improvements of coastal ocean predictions due to coupling between wave and hydrodynamic models.

The structure of the paper is as follows. The wave and hydrodynamic models and the processes of their interaction are described in Sect. 2. Section 3 addresses the effects of hydrodynamics on wave model performance, while in Sect. 4 we discuss the effects of waves on hydrodynamics and improvement of short-term forecast; followed finally by concluding remarks.

\section{Model description}

\subsection{Hydrodynamical model}

The General Estuarine Transport Model (GETM, Burchard and Bolding, 2002) was used in this study to simulate the circulation. This model solves the primitive equations for momentum, temperature, salinity and water level. The model setup described here uses the $k-\varepsilon$ turbulence closure to solve for the turbulent kinetic energy $k$ and its dissipation rate $\varepsilon$. Horizontal discretization was done on a spherical grid. The coarse resolution North Sea-Baltic Sea (3 nautical miles and $21 \sigma$ layers) outer model was described in more detail by Staneva et al. (2009); see also Fig. 1 for the maps of model domains. The sea surface elevation at the open boundary was generated using 13 tidal constituents obtained from the satellite altimetry via the OSU Tidal Inversion Software (Egbert and Erofeeva, 2002). The model was forced by atmospheric fluxes computed from bulk aerodynamic formulas. These formulas used model-simulated sea surface temperature, $2 \mathrm{~m}$ air temperature and relative humidity together with $10 \mathrm{~m}$ winds from atmospheric analysis data. This information was derived from the regional model COSMO-EU operated by the German Weather Service (DWD; Deutscher Wet- terdienst) with a horizontal resolution of $7 \mathrm{~km}$. River runoff data were provided by the German Federal Maritime and Hydrographic Agency (BSH; Bundesamt für Seeschifffahrt und Hydrographie). A setup for the German Bight based on the same model with about $1 \mathrm{~km}$ horizontal resolution was nested in the coarser domain model as explained by Staneva et al. (2009). Further downscaling to the scales of the Wadden Sea coastal areas was implemented in the nested area in the German Bight resolved with $200 \mathrm{~m}$ horizontal resolution. All model configurations account for flooding and drying, which is a fundamental dynamic process in the Wadden Sea.

\subsection{Wave model}

Wave model (WAM) is a third-generation wave model which solves the wave transport equation explicitly without any presumptions on the shape of the wave spectrum. The basic physics and numerics of the WAM Cycle 4 wave model, which is described in Komen et al. (1994) and Guenther et al. (1992) are kept in the new release WAM 4.5.3. In the coupled model system, the source function integration scheme of Hersbach and Janssen (1999) and the reformulated wave model dissipation source function (Bidlot et al., 2005), later reviewed by Bidlot et al. (2007) and Janssen (2008), are incorporated. Additionally, depth-induced wave breaking (Battjes and Janssen, 1978) has been included as source function. Depth and/or current fields can be non-stationary. It is crucial for strongly tidally forced shallow areas, like the German Bight, to allow model grid points to fall dry and account for refraction due to spatially varying current and depth. These modifications are of utmost importance for the improvement of wave modelling results in the coastal areas such as the Wadden Sea. The wave model code is freely available under http://mywave.github.io/WAM/.

Similar to the circulation model, the open boundary conditions for the German Bight WAM are taken from the regional WAM setup for the North Sea area (with a spatial resolution of ca. $5 \mathrm{~km}$ ). The German Bight wave model has the same horizontal resolution and uses the same topography as the circulation model GETM. The driving wind fields are the same as the ones used in the hydrodynamical model. The required boundary information at the open boundaries of the North Sea model is derived from the regional wave model EWAM for Europe that is running twice a day in the operational wave forecast routine of the DWD. Within the framework of Coastal Observing System for Northern and Arctic seas (COSYNA), a pre-operational wave and hydrodynamic forecast system has successfully been implemented and is running continuously since December 2009, providing hindcast and forecast data freely available on the COSYNA website under http://www.hzg.de/institutes_platforms/cosyna/index. php.en. 
Table 1. Statistics of the validation. In addition to mean and standard deviation, the coefficients of a linear regression are given.

\begin{tabular}{|c|c|c|c|c|c|c|c|c|}
\hline & \multicolumn{4}{|c|}{ Elbe } & \multicolumn{4}{|c|}{ Hoernum Tief } \\
\hline & \multicolumn{2}{|r|}{ Hs (m) } & \multicolumn{2}{|r|}{$\operatorname{tm} 1(\mathrm{~s})$} & \multicolumn{2}{|r|}{ Hs (m) } & \multicolumn{2}{|r|}{$\operatorname{tm} 1(\mathrm{~s})$} \\
\hline \multirow[t]{2}{*}{ Mean measurements } & & 1.10 & & 4.36 & & 0.33 & & 2.43 \\
\hline & WAM & WAM-GETM & WAM & WAM-GETM & WAM & WAM-GETM & WAM & WAM-GETM \\
\hline Bias & 0.004 & -0.025 & 0.245 & 0.174 & -0.073 & -0.120 & 0.326 & 0.150 \\
\hline SD & 0.164 & 0.171 & 0.439 & 0.397 & 0.117 & 0.136 & 0.350 & 0.293 \\
\hline Slope & 1.051 & 1.085 & 0.982 & 1.026 & 0.779 & 0.835 & 0.322 & 0.574 \\
\hline Intercept & -0.061 & -0.068 & -0.169 & -0.285 & 0.146 & 0.174 & 1.323 & 0.886 \\
\hline
\end{tabular}

Table 2. Elevation amplitude (cm) RMSE and mean errors (model-observations) for the coupled wave-circulation model and GETM-only model for the tide gauge data from British Oceanographic Data Centre (BODC) over the German Bight area.

\begin{tabular}{lrr|rr}
\hline & \multicolumn{2}{c|}{ RMSE } & \multicolumn{2}{c}{ Mean error } \\
\cline { 2 - 5 } & WAM-GETM & GETM & WAM-GETM & GETM \\
\hline Period 1 (1 December 2013-12 December 2013) & 12.4 & 19.4 & -7.6 & -11.5 \\
Period 2 (1 December 2013-5 December 2013) & 11.8 & 15.2 & -6.6 & -10.4 \\
Period 3 (6 December 2013-7 December 2013) & 13.6 & 22.7 & -8.5 & -18.5 \\
\hline
\end{tabular}

\subsection{Coupled model implementation and periods of analyses}

The original version of GETM was modified to account for the depth-dependent radiation stress and Stokes drift. The terms were calculated from the integrated wave parameters according to Mellor $(2008,2011)$ and Kumar et al. (2012). The gradients of the radiation stresses serve as additional explicit wave forcing in the momentum equations for the horizontal velocity components. Here the Stokes drift components have been subtracted from the wave processes in order to transfer them to the Eulerian framework. Moghimi et al. (2013) studied the effects of the two different approaches utilising the radiation stress (Mellor, 2011) and vortex force (Ardhuin et al., 2008) using WAM-GETM coupled models and showed that the results for the longshoredirected transport are similar for both formulations. Recently Aiki and Greatbatch (2013) showed that the radiation stress parameterization is applicable for small bottom slopes and Grashorn et al. (2015) showed that radiation stress formalism is applicable for shallow areas like the German coastal ones. They also demonstrated that the criterion proposed by Mellor (2013) to test the applicability of the radiation stress method gives reasonable results in this region. This gives us a confidence that, despite the known limitations of the radiation stress formalism it is well applicable for our study area. Additionally, the bottom friction modifications dependent upon bottom roughness and wave properties (Styles and Glenn, 2000) have been implemented. Turbulent kinetic energy due to wave friction (wave breaking/white capping and bottom dissipation) that is wave-enhanced turbulence has also been taken into consideration (Pleskachevsky et al., 2011).

In order to demonstrate the impact of wave-current interaction on coastal model simulations we performed two different experiments. In the first one the wave model WAM and the circulation model GETM have been run separately (we will further refer to it as the non-coupled run). The results have been compared with the WAM-GETM coupled model system, in which all wave-hydrodynamic processes described above are considered. We will further refer to it as the coupled model run. Three case studies have been analysed here, which we consider interesting in terms of both atmospheric conditions/extreme events and observational data availability.

The first analyses period is in July 2011, which was a calm weather period. Two different wind regimes were dominating the atmospheric state in July 2011, which will be addressed separately.

The next two analyses periods are chosen in order to address the effects of two of the most severe storm surges affecting our study region in the last hundred years. The first storm surge is the storm Britta of 31 October-1 November 2006 causing serious damages for the off-shore infrastructures and shipping in the North Sea region. Storm Britta was characterized by a deep low-pressure centre that moved on a trajectory from north of Scotland to western Norway and then eastwards through the Baltic Sea. Severe storm surge damages occurred in the east Wadden Sea. Extreme sea level during this storm surge is considered as a 100-year event (Madsen et al., 2007). In addition to the storm surge, unusually high waves have been measured in the southern North 
(a)

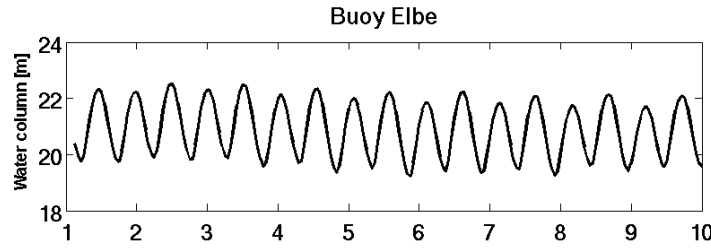

(b)

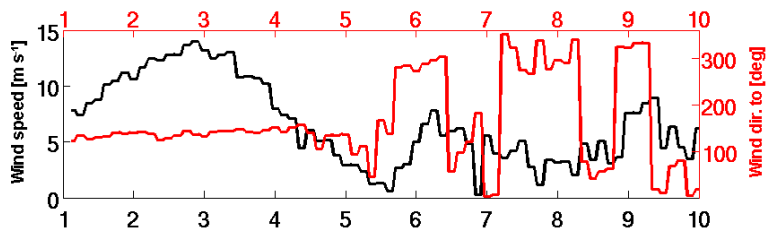

(c)

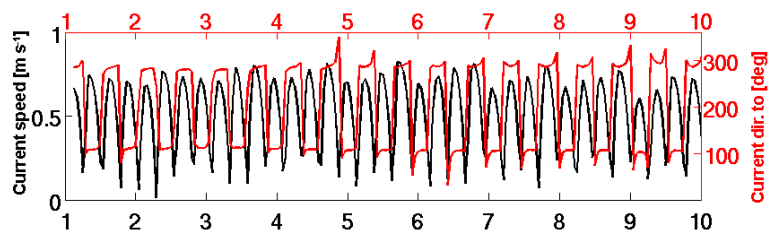

(d)

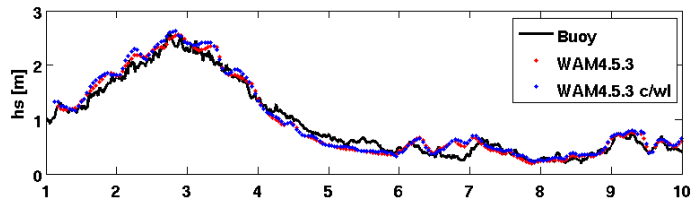

(e)

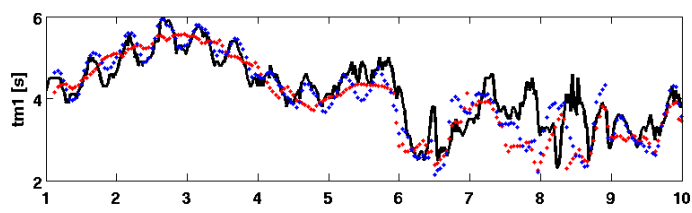

(f)

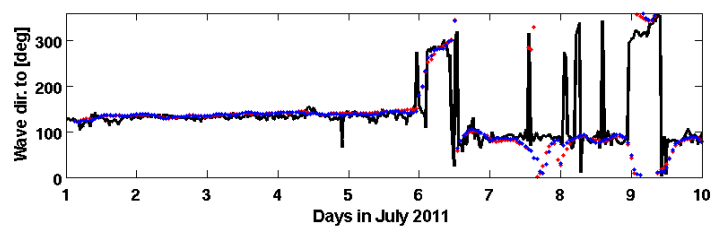

Figure 2. Time series at the buoy Elbe station (see Fig. 1 for its location) from 1 to 10 July 2011 of (a) water column (m), (b) wind speed $\left(\mathrm{m} \mathrm{s}^{-1}\right)$ (black line, left axis) and wind direction (deg.) (red line, left axis); (c) surface current magnitude (black line, left axis) and current direction (red line, left axis); (d) significant wave height (m); (e) mean period tm1 (s); and (f) wave direction (\%). For the patterns (d)-(f), the black line corresponds to the buoy measurements, red dots correspond to coupled model simulations, blue dots correspond to the wave-only model.

Sea developing on northern North Sea and propagated southward under the influence of strong north winds with a long fetch. The storm Britta has been given particular attention in our analyses for the types of changes that may occur during single event (Bartholomä et al., 2009; Lettmann et al., 2009; Stanev et al., 2011; Grashorn et al., 2015).

The second extreme event that we consider here is the winter storm Xavier on 5-6 December 2013, causing severe
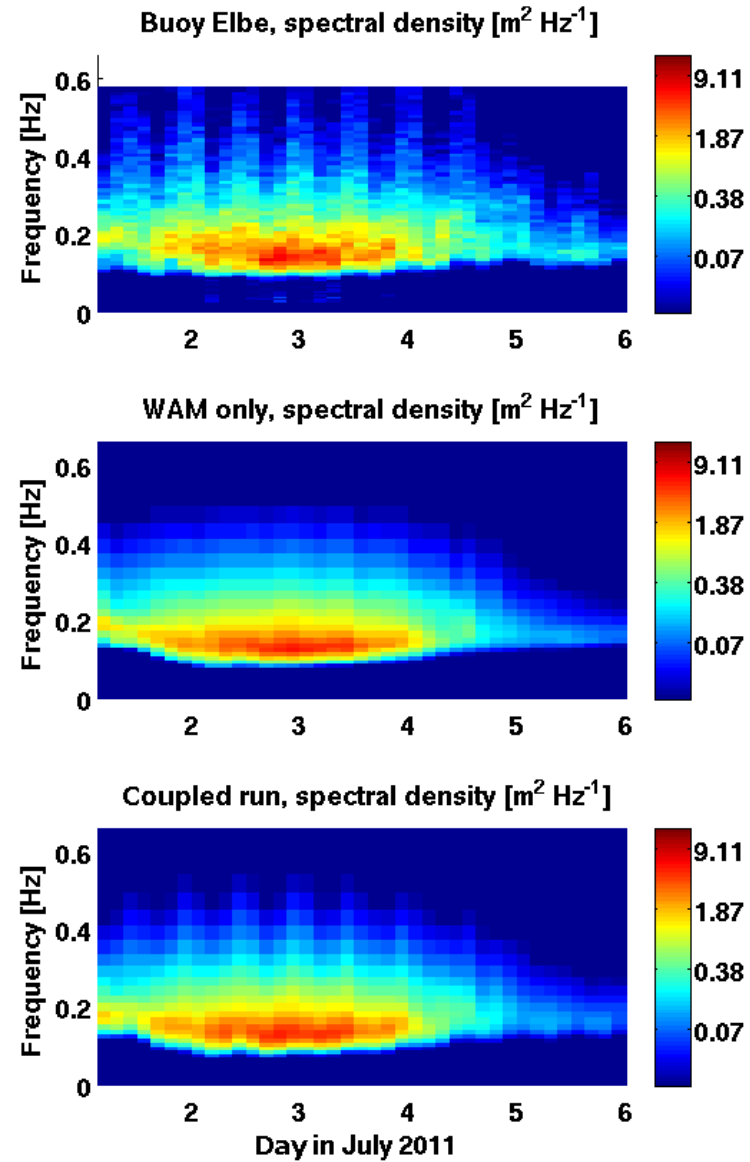

Figure 3. Comparison of measured (top) and computed values of the spectral energy density at the buoy Elbe (see Fig. 1 for its location).

flooding and devastation along the German North Sea coast. Besides extreme high water levels along the coasts, extreme sea state conditions have been observed causing serious erosion of dunes and sand displacements on the barrier islands.

\section{Impact of hydrodynamics on waves}

\subsection{Model validation}

At the buoy Elbe, which is located in the open sea (water depth about $21 \mathrm{~m}$, see the middle panel of Fig. 1), two different wind regimes occurred between 1 and 10 July 2011 (Fig. 2). From 1 to 5 July the dominating north-western wind did not change its direction (see the red line in Fig. 2b). However wind speed increased from $7.7 \mathrm{~m} \mathrm{~s}^{-1}$ on 1 July to a maximum of $15 \mathrm{~m} \mathrm{~s}^{-1}$ on 3 July (Fig. 2c). The decrease of wind speed to moderate values after 5 July 2011 (less than $5 \mathrm{~m} \mathrm{~s}^{-1}$ ) was accompanied by changing wind direction. The variations of water depth and currents are tidally dominated (Fig. 2a) and not much influenced by the wind during the whole period. The observed significant wave height (Fig. 2d) and the 

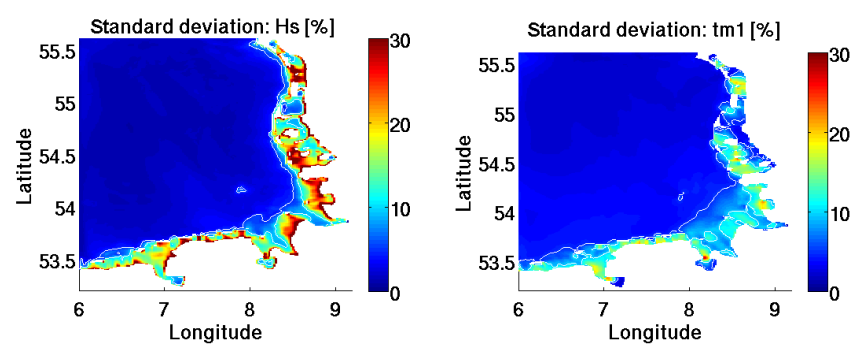

Figure 4. Impact of hydrodynamics on waves: normalized standard deviation (estimated as the difference between the control run and the coupled run relative to the control run values) of significant wave height (Hs, left) and mean period (tm1, right) between coupled wave-circulation model and wave-only model. Averaging is for 1 month (July 2011). The 5 and $10 \%$ isolines are plotted with white lines.

wave direction (Fig. 2f) are generally in a good agreement with the measurements for both the wave-only model and the coupled wave-circulation model. A clear tidal signal can be seen in the wave periods in the coupled model simulations, which accounted for the varying currents. It is noteworthy that in addition to current refraction, the tidal water level variations and depth refraction play a strong role in tidaldominated seas like the North Sea. This replicates the available measurements well (blue dots in Fig. 2e). Consequently, the difference of the standard deviation (SD) of the tm1 period decreases form $0.439 \mathrm{~s}$ in the non-coupled run to $0.397 \mathrm{~s}$ in the coupled one and the bias (model-measurement) decreases from 0.245 to $0.174 \mathrm{~s}$, respectively (see Table 1 ). The bias and SD of the significant wave height (Hs) are small in both runs, demonstrating that the wave models fit well with the observations.

The frequency wave spectra from the Elbe buoy and the two runs are shown in Fig. 3 for the first 5 days in July during the strong wind event. Similarly to Fig. 2, the patterns of wave spectra from the measurements and those of the coupled model run are in very good agreement (compare the top and bottom panel of Fig. 3). This is not the case for the noncoupled wave model (the middle panel in Fig. 3). The tidal currents are mainly affecting the tail of the spectra, whereas the energy around the peak is not much different in all three panels. The statistical analysis of the observations and simulations (see Table 1) clearly demonstrates the improvement of the quality of coupled wave-circulation model forecasts for the German Bight in comparison to the non-coupled model.

\subsection{Spatial patterns}

To quantify the impact of currents, including water depth hydrodynamics on the results of wave model, the SD of Hs and the mean period (tm1), of the coupled run normalized by the mean values of the non-coupled wave model are shown in Fig. 4. The horizontal patterns are given as a 1-month average for July 2011. In the open North Sea area there are no signif-
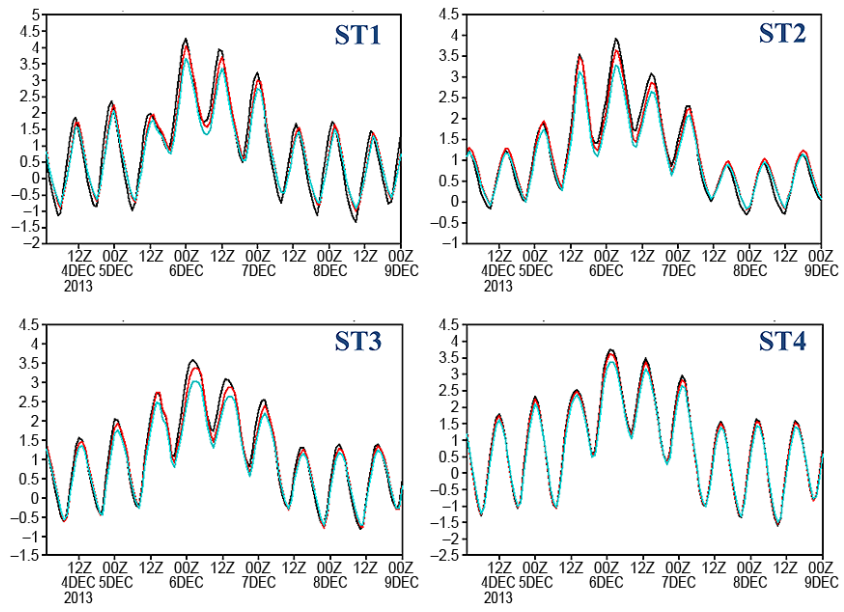

Figure 5. Time series of sea level elevation (SLE) in (m) at four coastal stations of the German Bight (ST1-ST4, see Fig. 1 for the locations). Black line corresponds to tide gauge observations, red line corresponds to the coupled wave-circulation model (WAMGETM) and green line corresponds to circulation-only model (GETM).
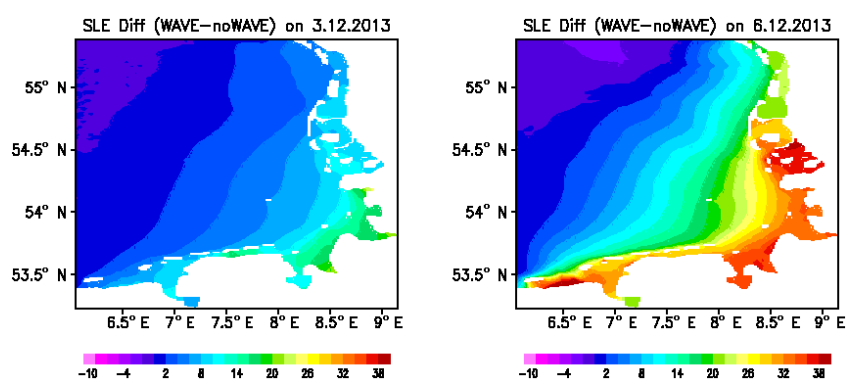

Figure 6. Sea level elevation (SLE) difference $(\mathrm{cm})$ between the coupled wave-circulation model (WAM-GETM) and circulationonly model (GETM) for the German Bight on 3 December 2013 at 01:00 UTC (left) and during the storm Xavier on 6 December 2013 at 01:00 UTC.

icant differences between the coupled and non-coupled wave modes for both Hs and tm1. However, along the coastal areas, where currents and water level change rapidly under the influence of tides, the impact of coupling seems to be significant. Within the German Bight coastal areas the SD of Hs goes up to $30 \%$, mainly due to the changes in water depth. The SD of tm 1 is about $10-15 \%$ in the coastal area. In particular, in the south-east of the German Bight, where the rivers Elbe and Weser are entering, the impact of coupling on the tm1 period spreads much further off-shore.

Interesting to notice are several relatively small areas, mainly located on the tidal inlets where the SD of tm1 reaches values of up to $30 \%$. These areas are characterized by strong currents, up to $1.5 \mathrm{~m} \mathrm{~s}^{-1}$ (see Staneva et al., 2009), often parallel to the waves inducing a large Doppler shift. The large SD in the entrance of the Jade Bay (lo- 

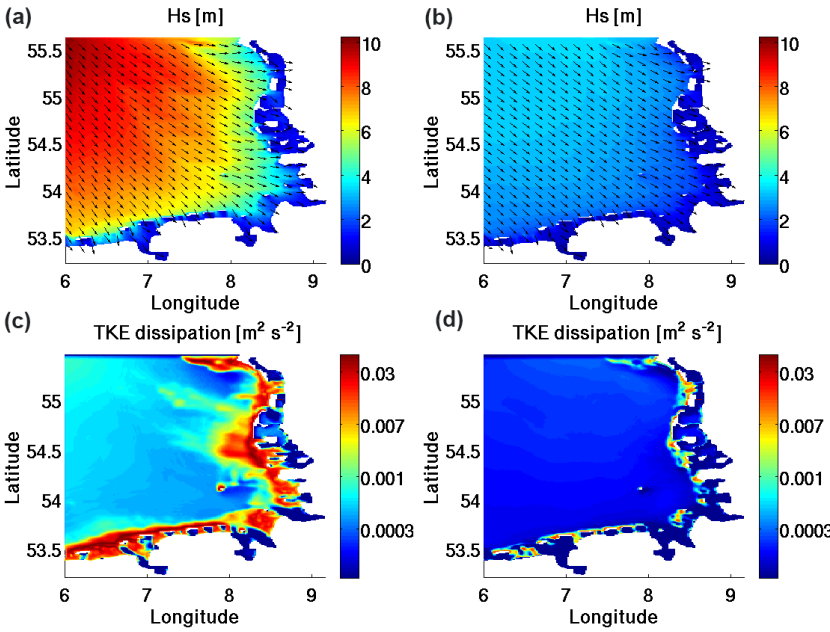

Figure 7. (a) Significant wave height $(\mathrm{m})$ in the German Bight during the peak of storm Britta on 1 November 2006 at 03:00 UTC. (b) Significant wave height $(\mathrm{m})$ in the German Bight during normal meteorological conditions on 3 November 2006 at 03:00 UTC. (c) TKE distribution in the German Bight during storm Britta on 1 November 2006 at 03:00 UTC. (d) TKE distribution in the German Bight during normal meteorological conditions on 3 November 2006 at 03:00 UTC.

cated in the east Wadden Sea which is the southern German Bight area with coordinates $8.25^{\circ} \mathrm{E}, 53.5^{\circ} \mathrm{N}$ and water depth $6 \mathrm{~m} \pm 1 \mathrm{~m}$ ) reveals that the wave variables $\mathrm{Hs}$ and $\mathrm{tm} 1$ increase substantially during northerly wind periods (inducing local wave growth, longer effective fetch) and opposing currents (responsible for wave blocking and Doppler shift).

\section{Impact of waves on hydrodynamics}

\subsection{Analyses for the periods of extreme events}

In this section we demonstrate the role of coupling by analysing the impact of waves on hydrodynamics during several extreme events. Sea level variability in four locations (ST1-ST4, see Fig. 1 for their geographical locations) are analysed along the German coast for the period including the extreme event Xavier on 6 December 2013 (see description in Sect. 2). The observations and simulations are shown in Fig. 5 for the tide gauge observations (black line), coupled wave-circulation model simulations (coupled run, red line) and the non-coupled run (circulation-only model, blue line). During normal meteorological conditions, the coupled and non-coupled models fit well with the tide gauge data. However, during the storm Xavier, the sea level predicted by the hydrodynamic-only model is underestimated with more than $40 \mathrm{~cm}$. It appears that the sea level predictions of the coupled model are closer to the measurements (compare the red and black lines). This demonstrates the importance of wave-current interactions also for the hydrodynamics. The root mean square errors (RMSEs) between observations and coupled model have been significantly reduced compared with the RMSE differences between the observations and circulation-only model for all coastal locations (Table 2). Predictions of storm events with coupled models could be of utmost importance for many coastal applications dealing with risk analyses (off-shore wind industry, oil platform operations, etc.) where higher accuracy is needed. This justifies the consideration of waves in operational forecasting.

\subsection{Spatial patterns}

In order to give an idea of the spatial distribution of the effects resulting from coupling, we show in Figure 6 the differences of sea surface elevation between the coupled and circulation-only models for 3 December 2013 at 01:00 UTC (normal meteorological situation, left panel) and 6 December 2013 at 01:00 UTC (extreme event, right panel). The waveinduced parameterization increases the average water level, which is more pronounced in the coastal area. In the open North Sea the effects of coupling are almost negligible. During normal conditions the difference of the sea level due to the coupling of circulation and wave models reaches a maximum of $10-15 \mathrm{~cm}$ in the area of the Elbe estuary. However, during the storm Xavier, the differences of simulated sea level when considering waves are more than $30 \mathrm{~cm}$ along the whole German coast. In some of the Wadden Sea areas the increase of water level in the simulations taking into consideration the wave-current interactions was above a half metre. The results shown here are indicative that the uncertainties in most of the presently used non-coupled operational models result from the missing non-linear feedback between strong tidal currents and wind waves. This can no longer be ignored in the operational oceanography, in particular in the coastal zone where the wave-circulation interplay seems to be dominant. The statistical analyses of simulated SLE vs. tide gauge data over the German Bight (Table 2) show that the coupling significantly improves the ocean predictions for the whole German coastal area. The RMSEs during the calm conditions are small in both coupled and circulation-only models. However during the extreme events the RMSE of sea surface elevation are significantly reduced when considering oceanwave interactions.

In the following we will demonstrate the effect of coupling on the storm Britta on 1 November 2011. During this storm event (see Fig. 7a), significant wave height over $10 \mathrm{~m}$ has been simulated in the open North Sea (close to the northwestern boundary). The southern North Sea area was exposed to waves with a magnitude of about 6-7 $\mathrm{m}$. Only 2 days later significant wave height dropped to $4 \mathrm{~m}$ within the German Bight (Fig. 7b). As an example of the impact of wave effects we show the dissipation of surface turbulent kinetic energy in the German Bight area at the peak of the storm at 03:00 UTC on 1 November (Fig. 7c) and under calm meteorological conditions (Fig. 7d). Along the coast dissipation 

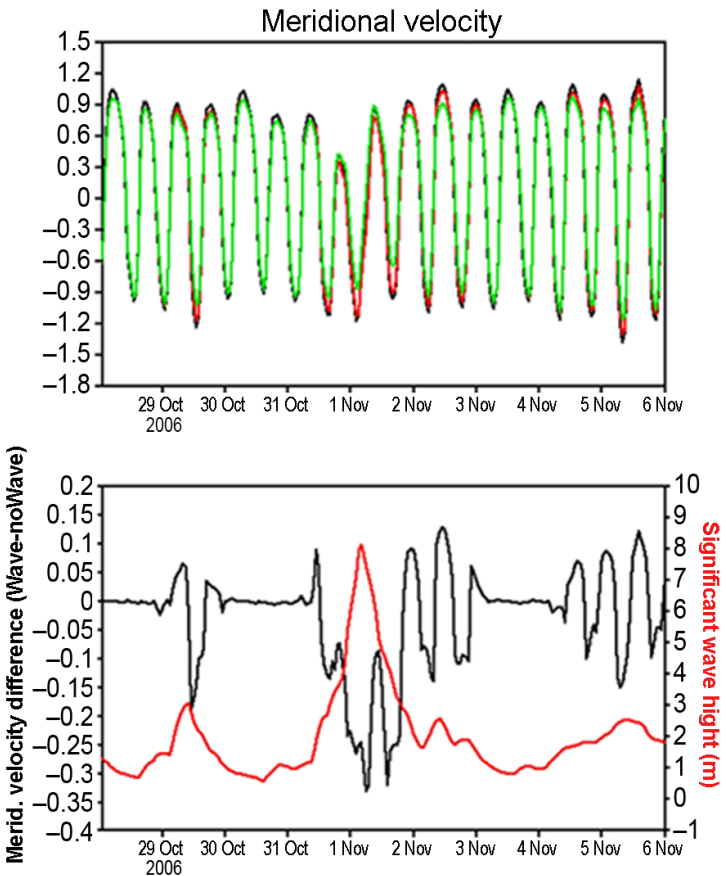
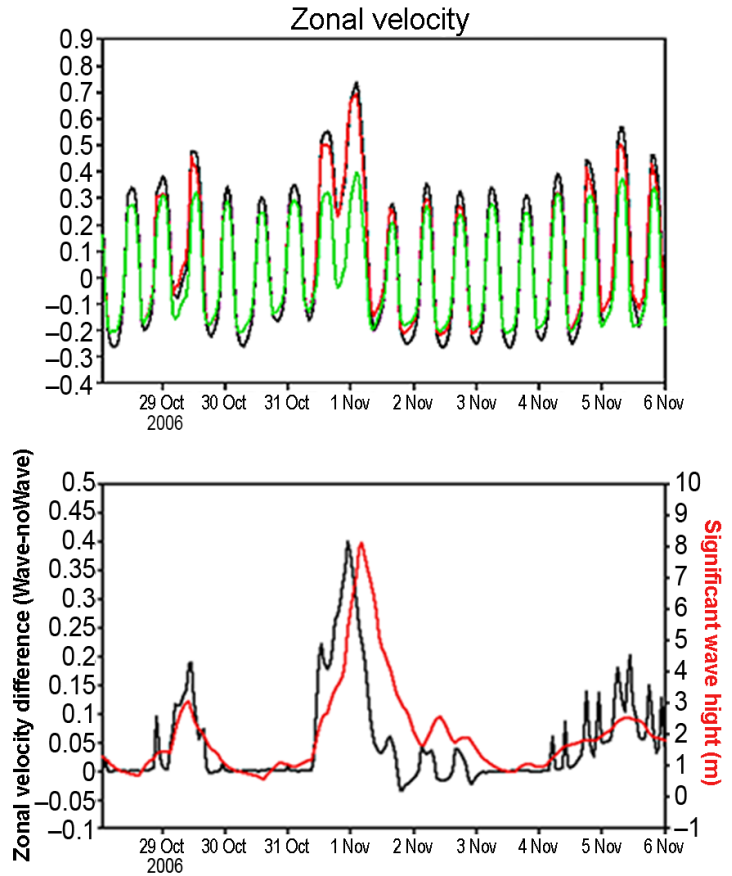

Figure 8. Top: meridional (left) and zonal (right) velocity time series $\left(\mathrm{m} \mathrm{s}^{-1}\right)$ on station W1 (see Fig. 1 for its location) from measurements (black line), coupled wave-circulation model (red line) and hydrodynamic-only model (green line) during storm Britta. Bottom: differences between the coupled and non-coupled model simulations of meridional (left) and zonal (right) velocity ( $\mathrm{m} \mathrm{s}^{-1}$ ) (black line) and significant wave height $(\mathrm{m})$ (red line).
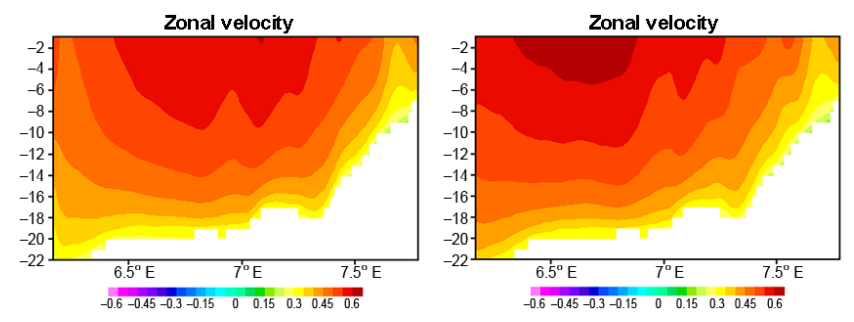

Figure 9. Zonal velocity vertical section $\left(\mathrm{m} \mathrm{s}^{-1}\right)$ during storm Britta on 1 November 2006 at 03:00 UTC (the location of the section is shown on Fig. 1) from the hydrodynamic-only model (left) and coupled model (right).

rates exceed $0.06 \mathrm{~m}^{2} \mathrm{~s}^{-2}$, which is about 100 times larger than under normal meteorological conditions.

Predictions of both zonal and meridional velocity have been also improved due to the coupling between the waves and circulation during storm Britta (see Fig. 8). The zonal velocity has been under-estimated in the circulation-only model (green line) and got closer to the ADCP data provided by the BSH for the coupled wave-circulation model (red line). There is also a very good correlation between the differences of the predicted velocity and significant wave height (Fig. 8, bottom patterns). During the storm Britta when the significant wave height reached almost $8 \mathrm{~m}$ in the coastal station the difference of the zonal velocity between the coupled run and the hydrodynamic model was more than $40 \mathrm{~cm} \mathrm{~s}^{-1}$. The transport along the coastal area has been also increased in the coupled runs (the differences of the zonal velocity between both runs being above $35 \mathrm{~cm} \mathrm{~s}^{-1}$ ). These results are indicative that coupled hydrodynamics and wave models could be of significant importance for further Lagrangian drift applications, e.g. for search and rescue operations as well as oilspill analyses. The effect of wave-current interactions on Lagrangian particle transport has been investigated in Röhrs et al. (2012, 2014).

The vertical section of the intensification of the longshore currents during the storm Britta is shown on Fig. 9 (the location of the section is plotted in Fig. 1). Not only does the longshore velocity increase but also its vertical structure has been changed through the effects of coupling. Similar behaviour has been also observed by Grashorn et al. (2015).

\section{Conclusions}

Wave and hydrodynamic hindcast and forecast for the North Sea and German Bight are of great importance for the management of coastal zones, ship navigation, off-shore wind energy, naval operations, etc. The storms and waves which they generate have direct impact on the coastal and marine environment. The population living in the coastal areas is recently concerned with the impacts of erosion and flooding, 
and actions aiming at better predictions, impact assessments of minimisation of damages are of greatest importance. Some driving forces that cause serious damages in coastal environments are due to the wave conditions. Their absolute and relative impact can be estimated by using coastal models. In this paper we demonstrated the improvements of coastal ocean predictions due to consideration of wave-current interaction for the North Sea and German Bight regions.

Improved forecast statistics once considering coupling is being demonstrated for both wave and circulation models. The coupled system presented here enables to provide reliable predictions as well as analyse long-term changes of wave and circulation conditions, including extreme events. The performance of the forecasting system was illustrated for the cases of several extreme events along with the effects of ocean waves on coastal circulation. It can be concluded that for our study area the use of radiation stress parameterization produced physically reasonable results. However, the different wave-induced formalisms lead to different limitations and thus no general recommendation should be performed. The improved skill resulting from the recent coupled model developments, in particular during storms, justifies further enhancements of the both forecast applications at operational services and long-term hindcasts and climate analyses for the North Sea and the German Bight.

Acknowledgements. This work was supported by EU FP7 Project MyOcean 2, Grant agreement 283367 and Horizon2020 Project MyOcean FO, Grant agreement 633085. The authors are thankful to W. Koch for preparing the model forcing and B. Gardeike for assistance with the graphics. We acknowledge the use of the measurement data provided by $\mathrm{BSH}$.

The article processing charges for this open-access publication were covered by a Research

Centre of the Helmholtz Association.

Edited by: H. Wehde

\section{References}

Aiki, H. and Greatbatch, R. J.: The vertical structure of the surface wave radiation stress for circulation over a sloping bottom as given by thickness-weighted-mean theory, J. Phys. Oceanogr., 43, 149-164, 2013.

Aiki, H. and Greatbatch, R. J.: A new expression for the form stress term in the vertically Lagrangian mean framework for the effect of surface waves on the upper-ocean circulation, J. Phys. Oceanogr., 44, 3-23, 2014.

Ardhuin, F., Rascle, N., and Belibassakis, K.: Explicit waveaveraged primitive equations using a generalized Lagrangian mean, Ocean Model., 20, 35-60, 2008.

Babanin, A. V., Chalikov, D., Young, I. R., and Savelyev, I.: Numerical and laboratory investigation of breaking of steep two- dimensional waves in deep water, J. Fluid Mech., 644, 433-463, 2010.

Bartholomä A., Kubicki A., Badewien T., and Flemming B. W.: Suspended sediment transport in the German Wadden Seaseasonal variations and extreme events, Ocean Dynam., 59, 213225, 2009.

Battjes, J. A. and Janssen, P.: Energy loss and setup due to breaking of random waves. International Conference on Coastal Engineering, ASCE, 569-587, 1978.

Bennis, A. and Ardhuin, F.: Comments on the depth-dependent current and wave interaction equations: a revision, J. Phys. Oceanogr., 41, 2008-2012, 2011.

Bidlot, J., Janssen P., and Abdalla S.: A revised formulation for ocean wave dissipation in CY29R1, Memorandum research department of ECMWF, 7 April 2005, File: R60.9/JB/0516, 2005.

Bidlot, J.-R., Janssen P., Abdalla S., and Hersbach H.:, A revised formulation of ocean wave dissipation and its model impact, ECMWF Tech. Memo. 509, Eur. Cent. for Medium-Range Weather Forecasting, Reading, UK, 2007.

Bolaños, R., Osuna, P., Wolf, J., Monabiu, J., and Sanchez-Arcilla, A.: Development of the POLCOMS-WAM current-wave model, Ocean Model., 36, 102-115, 2011.

Bolaños, R., Brown, J. M., and Souza, A. J.: Wave-current interactions in a tide dominated estuary, Cont. Shelf Res., 87, 109-123, doi:10.1016/j.csr.2014.05.009, 2014.

Breivik, O., Mogensen, K., Bidlot, J.-R., Balmaseda, M. A., and Janssen, P. A. E. M.: Surface wave effects in the NEMO ocean model: Forced and coupled experiments, J. Geophys. Res.Oceans, 120, 2973-2992, 2015.

Brown, J. M., Bolaños, R., and Wolf, J.: Impact assessment of advanced coupling features in a tide-surge-wave model, POLCOMS-WAM, in a shallow water application, J. Mar. Syst., 87, 13-24, 2011.

Brown, J. M., Bolaños, R., and Wolf, J.: The depth-varying response of coastal circulation and water levels to 2-D radiation stress when applied in a coupled wave-tide-surge modelling system during an extreme storm, Coast. Eng., 82, 102-113, 2013.

Burchard, H. and Bolding K.: GETM - a General Estuarine Transport Model, No EUR 20253 EN, printed in Italy, European Comission, 2002.

Egbert, G. D. and Erofeeva, S. Y.: Efficient inverse modeling of barotropic ocean tides, J. Atmos. Ocean. Technol. 19, 183-204, 2002.

Günther, H., Hasselmann, S., and Janssen, P. A. E. M.: The WAM Model Cycle 4.0. User Manual, Technical Report No. 4, Deutsches Klimarechenzentrum, Hamburg, Germany, 102 pp., 1992.

Grashorn, S., Lettmann, K. A., Wolff, J.-O., Badewien, T. H., and Stanev, E. V.: East Frisian Wadden Wea hydrodynamics and wave effects in an unstructured-grid model, Ocean Dynam., 65, 419434, 2015.

Hersbach, H. and Janssen P.: Improvements of the short fetch behaviour in the WAM model, J. Atmos. Ocean. Technol., 16, 884892, 1999.

Janssen, P: Ocean wave effects on the daily cycle in SST, J. Geophys. Res., 117, C00J32, doi:10.1029/2012JC007943, 2012.

Komen, G. J., Cavaleri, L., Donelan, M., Hasselmann, K., Hasselmann, S., and Janssen, P.: Dynamics and modelling of ocean 
waves, Cambridge University Press, Cambridge, UK, 560 pp., 1994.

Kumar, N., Voulgaris, G., Warner, J. C., and Olabarrieta, M.: Implementation of the vortex force formalism in the coupled oceanatmosphere-wave-sediment transport (COAWST) modelling system for inner shelf and surf zone applications, Ocean Model., 47, 65-95, 2012.

Lane, E. M., Restrepo, J. M., and McWilliams, J. C.: Wave-current interaction: a comparison of radiation-stress and vortex-force representations, J. Phys. Oceanogr., 37, 1122-1141, 2007.

Lettmann, K., Wolff, J.-O., and Badewien, T.: Modeling the impact of wind and waves on suspended particulate matter fluxes in the East Frisian Wadden Sea (southern North Sea), Ocean Dynam., 59, 239-262, 2009.

Lin, R. Q. and Perrie, W.: Wave-current interactions in an idealized tidal estuary, J. Geophys. Res., 108, 1-18, doi:10.1029/2001JC001006, 2003.

Longuet-Higgins M. S. and Stewart R. W.: Radiation stresses in water waves: a physical discussion with applications, Deep-Sea Res., 11, 529-562, 1964.

Madsen, K. S., Hoyer, J. L., and Tschering, C. C.: Near-coastal satellite altimetry: sea surface 15 height variability in the North Sea-Baltic Sea area, Geophys. Res. Lett., 34, L14601, doi:10.1029/2007GL029965, 2007.

McWilliams, J., Restrepo, J., and Lane, E.: An asymptotic theory for the interaction of waves and currents in coastal waters, J. Fluid Mech., 511, 135-178, 2004.

Mellor, G.: The three-dimensional current and surface wave equations, J. Phys. Oceanogr., 33, 1978-1989, 2003.

Mellor, G.: Some consequences of the three-dimensional current and surface equations, J. Phys. Oceanogr., 35, 2291-2298, 2005.

Mellor, G.: The depth-dependent current and wave interaction equations: a revision, J. Phys. Oceanogr., 38, 2587-2596, 2008.

Mellor, G.: Wave radiation stress, Ocean Dynam., 61, 563-568, 2011.

Mellor, G.: Waves, circulation and vertical dependance, Ocean Dynam., 63, 447-457, 2013.

Michaud, H., Marsaleix, P., Leredde, Y., Estournel, C., Bourrin, F., Lyard, F., Mayet, C., and Ardhuin, F.: Three-dimensional modelling of wave-induced current from the surf zone to the inner shelf, Ocean Sci., 8, 657-681, doi:10.5194/os-8-657-2012, 2012.

Moghimi, S., Klingbeil, K., Gräwe, U., and Burchard, H.: A direct comparison of a depth-dependent radiation stress formulation and a vortex force formulation within a three-dimensional coastal ocean model, Ocean Model., 70, 132-144, 2013.

Pleskachevsky, A., Dobrynin, M., Babanin, A. V., Günther, H., and Stanev, E.: Turbulent mixing due to surface waves indicated by remote sensing of suspended particulate matter and its implementation into coupled modeling of waves, turbulence and circulation, J. Phys. Oceanogr., 41, 708-724, doi:10.1175/2010JPO4328.1, 2011.
Polton, J. A., Lewis, D. M., and Belcher, S. E.: The Role of WaveInduced Coriolis-Stokes Forcing on the Wind-Driven Mixed Layer, J. Phys. Oceanogr., 35, 444-457, doi:10.1175/JPO2701.1, 2005.

Prandle D., Hargreaves J. C., McManus J. P., Campbell A. R., Duwe K., Lane A., Mahnke P., Shimwell S., and Wolf J.: Tide, wave and suspended sediment modelling on an open coast, Coast. Eng., 4, 237-267, 2000.

Röhrs, J., Christensen, K. H., Hole, L. R., Broström, G., Drivdal, M., and Sundby, S.: Observation-based evaluation of surface wave effects on currents and trajectory fore-casts, Ocean Dynam., 62, 1519-1533, 2012.

Röhrs, J., Christensen, K. H., Vikebo, F., Sundby, S., Saetra, O., and Broström, G.: Wave- induced transport and vertical mixing of pelagic eggs and larvae, Limnol. Oceanogr., 59, 1213-1227, 2014.

Roland, A. and Ardhuin, F.: On the developments of spectral wave models: numerics and parameterizations for the coastal ocean, Ocean Dynam., 64, 833-846, 2014.

Roland, A., Cucco, A., Ferrarin, C., Hsu, T., Liau, J., Ou, S., Umgiesser, G., and Zanke, U.: On the development and verification of a 2-D coupled wave-current model on unstructured meshes, J. Mar. Syst., 78, 244-254, 2009.

Stanev, E. V., Schulz-Stellenfleth, J., Staneva, J., Grayek, S., Seemann, J., and Petersen, W.: Coastal observing and forecasting system for the German Bight - estimates of hydrophysical states, Ocean Sci., 7, 569-583, doi:10.5194/os-7-569-2011, 2011.

Staneva, J., Wolff, J.-O., Badewien, T., Reuter, R., Flemming, B., Bartholomae, A., and Bolding, K.: Hydrodynamics and sediment dynamics in the German Bight, A focus on observations and numerical modeling in the East Frisian Wadden Sea, Cont. Shelf Res., 29, 302-319, 2009.

Styles, R. and Glenn, S.: Modeling stratified wave and current bottom boundary layers on the continental shelf, J. Geophys. Res., 105, 24119-24124, 2000.

Uchiyama, Y., McWilliams, J., and Shchepetkin, A.: Wave-current interaction in an oceanic circulation model with a vortex-force formalism: application to the surf zone, Ocean Model., 34, 1635,2010 .

Weber, J. E. H., Brostrom, G., and Saetra, O.: Eulerian versus Lagrangian approaches to the wave-induced transport in the upper ocean, J. Phys. Oceanogr., 31, 2106-2118, 2006.

Wolf, J. and Prandle, D.: Some observations of wave-current interaction, Coast. Eng., 37, 471-485, 1999.

Wolf, J., Brown, J. M., Bolaños, R., and Hedges, T.: Waves in coastal and estuarine waters, in: Treatise on Estuarine and Coastal Science, edited by: Wolanski, E. and McLusky, D., Elsevier, 2, 171-212, 2011.

Zodiatis, G., Galanis, G., Kallos, G., Nikolaidis, A., Kalogeri, C., and Liakatas, S. S.: The impact of sea surface currents in wave power potential modeling, Ocean Dynam., 65, 1547-1565, 2015. 